\title{
NOTE ON THERMODYNAMIC SURFACES
}

BY J. E. TREVOR

\section{Conditions of stability}

In his investigation of the energy surface for a one-component system, ${ }^{x}$ Gibbs has given an ingenious and convincing demonstration of the theorem that, when $p, \theta$ are the pressure and absolute temperature of a thermodynamic system in a state of dissipated energy determined by the volume $v$ and the entropy $\eta$, and $v+\delta v, \eta+\delta \eta$ is another state, not a state of dissipated energy at $p, \theta$, it is true that

$$
\delta e+p \delta v-\theta \delta \eta>0 .
$$

Otherwise stated, in all variations of state imagined as occurring from a state of stable equilibrium at $p, \theta$ to a state not in stable equilibrium at $p, \theta$,

$$
\delta e>-p \delta v+\theta \delta \eta .
$$

It is possible, however, to have a succession of univariant states or of invariant states that are all states of stable equilibrium at $p, \theta$. In such a case, no one state of the succession will spontaneously pass into another, the equilibrium of each : 'ate will be 'neutral'. If the change through the succession of stable states be reversibly effected, Gibbs's demonstratic in yields

$$
\delta e=-p \delta v+\theta \delta \eta \text {. }
$$

For a state to be one of stable equilibrium at the piessure and temperature $p, \theta$, it is therefore necessary that, in $n 1$ variations of the state,

$$
\delta e \geqq-p \delta v+\theta \delta \eta
$$

On passing from any point $v, \eta$ on the ergy surface

$$
e=e(v, \eta)
$$

for one-phase states, or for states of $(w$, given phases, or of three given phases, to an adjacent poir. $\sim r \delta v, \eta+\delta \eta$ on the surface,

1 Trans. Conn. Acad. 2, $382(1873)$. 
84

$$
\begin{gathered}
\text { J. E. Trevor } \\
\delta e=\frac{\partial e}{\partial v} \delta v+\frac{\partial e}{\partial \eta} \delta \eta \\
+\frac{\mathrm{I}}{2}\left(\frac{\partial^{2} e}{\partial v^{2}}(\delta v)^{2}+2 \frac{\partial^{2} e}{\partial v \partial \eta} \delta v \delta \eta+\frac{\partial^{2} e}{\partial \eta^{2}}(\delta \eta)^{2}\right)+\cdots ;
\end{gathered}
$$

whence, because of the above criterion of stability, which may be written

$$
\delta e \geqq \frac{\partial e}{\partial v} \delta v+\frac{\partial e}{\partial \eta} \delta \eta
$$

it appears that the condition that the point $v, \eta$, shall represent a state of equilibrium is

$$
\frac{\partial^{2} e}{\partial v^{2}}(\delta v)^{2}+2 \frac{\partial^{2} e}{\partial v \partial \eta} \delta v \delta \eta+\frac{\partial^{2} e}{\partial \eta^{2}}(\delta \eta)^{2} \geqq 0
$$

for all values of $v, \eta$. This condition expresses that no portion of the surface may lie below the tangent plane passing through the point $v, \eta$. It expresses, in particular, that a succession of states in neutral equilibrium is represented by the points on a line common to the surface and the plane tangent thereto at the point $v, \eta$; and that, at a point representing a state of stable equilibrium that is not also a state of netitral equilibrium, the surface is convex downward in all directions. All portions of the surface that do not satisfy these conditions must represent thermodynamically instable states.

When

$$
e=e(v, \eta)
$$

is the equation of the continuous succession of stable and instable one-phase states, a point representing a state of stable equilibrium must satisfy the condition

$$
\frac{\partial^{2} e}{\partial v^{2}}(\delta v)^{2}+2 \frac{\partial^{2} e}{\partial v \partial \eta} \delta v \delta \eta+\frac{\partial^{2} e}{\partial \eta^{2}}(\delta \eta)^{2}>0 .
$$

The necessary and sufficient conditions for this quadratic expression to be positive, i. e., for the surface to be convex downward in every direction at $v, \eta$, are

$$
\mathrm{H}(e)>0, \quad \quad \partial^{2} e / \partial v^{2}>0,
$$

where $\mathrm{H}(e)$ denotes the hessian of the function $e(v, \eta)$. A con- 
sequence of these 'conditions of stability' is the further inequality

$$
\frac{\partial^{2} e}{\partial \eta^{2}}>0
$$

\section{A problem}

It was further pointed ont by Gibbs that the thermodynamic properties represented by the surface

$$
e=e(v, \eta)
$$

are represented by the surfaces

$$
\begin{aligned}
& f=f(v, \theta) \\
& g=g(p, \eta) \\
& h=h(p, \theta) ;
\end{aligned}
$$

the functions $f, g, h$ being defined by the equations

$$
\begin{aligned}
& f(v, \theta)=e-\theta_{\eta} \\
& g(p, \eta)=e+p v \\
& h(p, \theta)=e+p v-\theta \eta ;
\end{aligned}
$$

wherefore we have the set of differential equations

$$
\begin{aligned}
& d e=-p d v+\theta d \eta \\
& d f=-p d v-\eta d \theta \\
& d g=v d p+\theta d \eta \\
& d h=v d p-\eta d \theta
\end{aligned}
$$

We may now seek to determine the curvatures of those portions of the $f, g, h$ surfaces that represent one-phase stable states, i. e., that correspond to the convex portions of the primitive energy surface.

\section{Auxiliary equations}

In this search we shall require to use a series of equations, which shall now be assembled for reference. We have

$$
\begin{aligned}
-d p & =\frac{\partial^{2} e}{\partial v^{2}} d v+\frac{\partial^{2} e}{\partial v \partial \eta} d \eta \\
d \theta & =\frac{\partial^{2} e}{\partial v \partial \eta} d v+\frac{\partial^{2} e}{\partial \eta^{2}} d \eta ;
\end{aligned}
$$

whence, by successive elimination of $d \eta$ and $d v$, 


$$
\mathrm{H}(e) \cdot d v=\left|\begin{array}{rr}
-d p & \frac{\partial^{2} e}{\partial v \partial \eta} \\
d \theta & \frac{\partial^{2} e}{\partial \eta^{2}}
\end{array}\right|, \quad \mathrm{H}(e) \cdot d \eta=\left|\begin{array}{cc}
\frac{\partial^{2} e}{\partial v^{2}} & -d p \\
\frac{\partial^{2} e}{\partial v \partial \eta} & d \theta
\end{array}\right|
$$

whence follow the desired equations,

$$
\begin{array}{ll|l}
\text { (a) }\left(\frac{\partial v}{\partial p}\right)_{\theta}=\frac{-\mathrm{I}}{\mathrm{H}(e)} \frac{\partial^{2} e}{\partial \eta^{2}} & \left(\frac{\partial \eta}{\partial \theta}\right)_{p}=\frac{\mathrm{I}}{\mathrm{H}(e)} \frac{\partial^{2} e}{\partial v^{2}} \\
\text { (b) } \quad\left(\frac{\partial v}{\partial \theta}\right)_{p}=\frac{-\mathrm{I}}{\mathrm{H}(e)} \frac{\partial^{2} e}{\partial v \partial \eta} & \left(\frac{\partial \eta}{\partial p}\right)_{\theta}=\frac{\mathrm{I}}{\mathrm{H}(e)} \frac{\partial^{2} e}{\partial v \partial \eta}
\end{array}
$$

and from these, by division,

$$
\text { (e) }\left(\frac{\partial v}{\partial \eta}\right)_{\theta}=-\frac{\partial^{2} e}{\partial \eta^{2}}: \frac{\partial^{2} e}{\partial v \partial \eta} \quad \mid \quad\left(\frac{\partial \eta}{\partial v}\right)_{p}=-\frac{\partial^{2} e}{\partial v^{2}}: \frac{\partial^{2} e}{\partial v \partial \eta} .
$$

The second derivatives

We now require to determine the signs of the second derivatives, and of the hessian, of each of the functions $f, g, h$ at points representing stable one-phase states.

For $\partial^{2} f \partial v^{2}$, we have

$$
\begin{aligned}
\frac{\partial f}{\partial v} & =-p \\
\frac{\partial^{2} f}{\partial v^{2}} & =-\left(\frac{\partial p}{\partial v}\right)_{\theta} \\
& =\mathrm{H}(e): \frac{\partial^{2} e}{\partial \eta^{2}} \\
& >0,
\end{aligned}
$$

by equation $(\alpha)$.

For $\partial^{2} r / \partial \theta^{2}$, we have

$$
\begin{aligned}
\frac{\partial f}{\partial \theta} & =-\eta \\
\frac{\partial^{2} f}{\partial \theta^{2}} & =-\left(\frac{\partial \eta}{\partial \theta}\right)_{v} \\
& =-\mathrm{I}:\left(\frac{\partial \theta}{\partial \eta}\right)_{v} \\
& =-\mathrm{I}: \frac{\partial^{2} e}{\partial \eta^{2}} \\
& <0 .
\end{aligned}
$$


For $\mathrm{H}(f)$, we have

$$
\begin{aligned}
-\frac{\partial^{2} f}{\partial v \partial \theta} & =-\left(\frac{\partial p}{\partial \theta}\right)_{v} \\
& =-\left(\frac{\partial p}{\partial \eta}\right)_{v}:\left(\frac{\partial \theta}{\partial \eta}\right)_{v} \\
& =\frac{\partial^{2} e}{\partial v \partial \eta}: \frac{\partial^{2} e}{\partial \eta^{2}}
\end{aligned}
$$

Hence we find

$$
\begin{aligned}
\mathrm{H}(f) & =\frac{\partial^{2} f}{\partial v^{2}} \frac{\partial^{2} f}{\partial \theta^{2}}-\left(\frac{\partial^{2} f}{\partial v \partial \theta}\right)^{2} \\
& =\frac{\frac{\partial^{2} e}{\partial v^{2}} \frac{\partial^{2} e}{\partial \eta^{2}}-\left(\frac{\partial^{2} e}{\partial v \partial \eta}\right)^{2}}{\frac{\partial^{2} e}{\partial \eta^{2}}} \cdot \frac{-\mathrm{I}}{\frac{\partial^{2} e}{\partial \eta^{2}}}-\frac{\left(\frac{\partial^{2} e}{\partial v \partial \eta}\right)^{2}}{\left(\frac{\partial^{2} e}{\partial \eta^{2}}\right)^{2}} \\
& =-\frac{\partial^{2} e}{\partial v^{2}}: \frac{\partial^{2} e}{\partial \eta^{2}} \\
& <o .
\end{aligned}
$$

For $\partial^{2} g / \partial p^{2}$, we have

$$
\begin{aligned}
\frac{\partial g}{\partial p} & ==v \\
\frac{\partial^{2} g}{\partial p^{2}} & =\left(\frac{\partial v}{\partial p}\right)_{\eta} \\
& =\mathrm{I}:\left(\frac{\partial p}{\partial v}\right)_{\eta} \\
& =-\mathrm{I}: \frac{\partial^{2} e}{\partial v^{2}} \\
& <0 .
\end{aligned}
$$

For $\partial^{2} g / \partial \eta^{2}$, we have

by equation $(c)$.

$$
\begin{aligned}
\frac{\partial g}{\partial \eta} & =\theta \\
\frac{\partial^{2} g}{\partial \eta^{2}} & =\left(\frac{\partial \theta}{\partial \eta}\right)_{p} \\
& =(\mathrm{H}) e: \frac{\partial^{2} e}{\partial v^{2}} \\
& >0
\end{aligned}
$$


For $\mathrm{H}(g)$, we have

$$
\begin{aligned}
\frac{\partial^{2} g}{\partial p \partial \eta} & =\left(\frac{\partial v}{\partial \eta}\right)_{p} \\
& =-\frac{\partial^{2} e}{\partial v \partial \eta}: \frac{\partial^{2} e}{\partial v^{2}},
\end{aligned}
$$

by equation $(f)$. Hence we have

$$
\begin{aligned}
\mathrm{H}(g) & =\frac{\partial^{2} g}{\partial p^{2}} \frac{\partial^{2} g}{\partial \eta^{2}}-\left(\frac{\partial^{2} g}{\partial p \partial \eta}\right)^{2} \\
& =\frac{-\mathrm{I}}{\frac{\partial^{2} e}{\partial v^{2}}} \cdot \frac{\frac{\partial^{2} e}{\partial v^{2}} \frac{\partial^{2} e}{\partial \eta^{2}}-\left(\frac{\partial^{2} e}{\partial v \partial \eta}\right)^{2}}{\frac{\partial^{2} e}{\partial v^{2}}}-\frac{\left(\frac{\partial^{2} e}{\partial v \partial \eta}\right)^{2}}{\left(\frac{\partial^{2} e}{\partial v^{2}}\right)^{2}} \\
& =-\frac{\partial^{2} e}{\partial \eta^{2}}: \frac{\partial^{2} e}{\partial v^{2}} \\
& <0 .
\end{aligned}
$$

For $\partial^{2} h / \partial p^{2}$, we have

$$
\begin{aligned}
\frac{\partial h}{\partial p} & =v \\
\frac{\partial^{2} h}{\partial p^{2}} & ==\left(\frac{\partial v}{\partial p}\right)_{\theta} \\
& =\frac{-1}{\mathrm{H}(e)} \frac{\partial^{2} e}{\partial \eta^{2}} \\
& <0,
\end{aligned}
$$

by equation $(a)$.

For $\partial^{2} h / \partial \theta^{2}$, we have

$$
\begin{aligned}
\frac{\partial h}{\partial \theta} & =-\eta \\
\frac{\partial^{2} h}{\partial \theta^{2}} & =-\left(\frac{\partial \eta}{\partial \theta}\right)_{p} \\
& =\frac{-1}{\mathrm{H}(e)} \frac{\partial^{2} e}{\partial v^{2}} \\
& <\mathrm{o},
\end{aligned}
$$

by equation (c). 
For $\mathrm{H}(h)$, we have

$$
\begin{aligned}
\frac{\partial^{2} h}{\partial \rho \partial \theta} & =\left(\frac{\partial v}{\partial \theta}\right)_{p} \\
& =\frac{-\mathrm{I}}{\mathrm{H}(e)} \frac{\partial^{2} e}{\partial v \partial \eta},
\end{aligned}
$$

by equation (b). Hence we find

$$
\begin{aligned}
\mathrm{H}(h) & =\frac{\partial^{2} h}{\partial p^{2}} \frac{\partial^{2} h}{\partial \theta^{2}}-\left(\frac{\partial^{2} h}{\partial p \partial \theta}\right)^{2} \\
& =\frac{-\frac{\partial^{2} e}{\partial \eta^{2}}}{\mathrm{H}(e)} \cdot \frac{-\frac{\partial^{2} e}{\partial v^{2}}}{\mathrm{H}(e)}-\frac{\left(\frac{\partial^{2} e}{\partial v^{2} \partial}\right)^{2}}{\mathrm{H}^{2}(e)} \\
& =\mathrm{I}: \mathrm{H}(e) \\
& >0 .
\end{aligned}
$$

These results are assembled in the following table:

$$
\begin{array}{lll}
\mathrm{H}(e)>0 & \frac{\partial^{2} e}{\partial v^{2}}>0 & \frac{\partial^{2} e}{\partial \eta^{2}}>0 \\
\mathrm{H}(f)<0 & \frac{\partial^{2} f}{\partial v^{2}}>0 & \frac{\partial^{2} f}{\partial \theta^{2}}<0 \\
\mathrm{H}(g)<0 & \frac{\partial^{2} g}{\partial p^{2}}<0 & \frac{\partial^{2} g}{\partial \eta^{2}}>0 \\
\mathrm{H}(h)>0 & \frac{\partial^{2} h}{\partial p^{2}}<0 & \frac{\partial^{2} h}{\partial \theta^{2}}<0
\end{array}
$$

\section{The curvatures}

From these data it appears that the portions of the $e, f, g, h$ surfaces that represent stable one-phase states are convex in the case of the $e$-surface, concave in the case of the $h$-surface, and saddle-shaped in the other two cases. In particular, the $f$-surface is convex at $v, \theta$ in the section parallel to $v$, and concave in the section parallel to $\theta$; and the $g$-surface is convex at $p, \eta$ in the section parallel to $\eta$, and concave in the section parallel to $p$.

It may be added that, whenever the primitive $e$-surface becomes concave at $v, \eta$, every curvature stated above will be changed into its opposite; and that, whenever the $e$-surface be- 
comes convex in one coordinate section and concave in the other, the $h$-surface will be so also, while the $f$-surface and the $g$-surface will become dome-shaped. For when the hessian of a surface $z=z(x, y)$ is positive the surface is dome-shaped, and is convex or concave according as

$$
\frac{\partial^{2} z}{\partial x^{2}} \quad \text { and } \quad \frac{\partial^{2} z}{\partial y^{2}}
$$

are both positive or both negative. When the hessian is positive these derivatives must have the same sign.

\section{Reciprocal character of the surfaces}

It may be noted in the above that the hessians of the $e$ and $h$ surfaces, and of the $f$ and $g$ surfaces, are the reciprocals of each other; that

$$
\begin{aligned}
& \mathrm{H}(e) \cdot \mathrm{H}(h)=\mathrm{I} \\
& \mathrm{H}(f) \cdot \mathrm{H}(g)=\mathrm{I} .
\end{aligned}
$$

This is one aspect of the reciprocal relation between the $e$ and $h$ and the $f$ and $g$ surfaces, which appears most clearly when it is noted that the ordinate of a point in either surface of either pair appears in the other surface as the segment

$$
z-\frac{\partial z}{\partial x} x-\frac{\partial z}{\partial y} y
$$

intercepted on the vertical axis by the tangent plane at the point; and that the horizontal coordinates of the one surface are the slopes of the other ${ }^{-}$(the changes of sign being due to taking $+p$ instead of $-p$ as a coordinate in two cases). This is exhibited in the tabulation:

$\begin{array}{cccc}\text { Ordinate } & \text { Intercept } & \text { Coordinates } & \text { Slopes } \\ e & h & v, \eta & -p, \theta \\ h & e & p, \theta & v,-\eta \\ f & g & v, \theta & -p,-\eta \\ g & f & p, \eta & v, \theta\end{array}$
Gibbs.

${ }^{1}$ With respect to the $e$ and $h$ surfaces, this has been pointed out by 
This reciprocity appears again in the relation

$$
e+h=f+g,
$$

which follows from the definition equations

$$
\begin{aligned}
& e=e \\
& f=e-\theta \eta \\
& h=e+p v-\theta \eta \text {. } \\
& g=e+p v \text {. }
\end{aligned}
$$

Cornell University. 